\title{
Distance perception as a function of photographic area of view
}

\author{
ROBERT N. KRAFT \\ Otterbein College, Westerville, Ohio \\ and \\ JEFFREY S. GREEN \\ Grinnell College, Grinnell, Iowa
}

\begin{abstract}
Distance perception of depicted objects was examined as a function of photographic area of view. Subjects viewed slides of natural outdoor scenes and directly estimated the distances to specified objects. Area of view was manipulated by means of photographing each scene with lenses of five different focal lengths: $135,80,48,28$, and $17 \mathrm{~mm}$. Distance perception along the pictorial depth plane was systematically transformed through changing the photographic area of view: the shorter the focal length of the camera lens, the wider the area of view, and the greater the perceived distance. Linear functions for each subject's distance judgments revealed a very high goodness of fit. Both the $y$-intercepts and the slopes increased as focal length decreased. The increasing $y$-intercepts suggest that viewers place themselves farther away from the depicted scene as focal length decreases, compensating for the advancing proximal edge. The increasing slopes suggest that distance information throughout the pictorial depth plane appears to change with focal length. The subjects also made direct judgments of foreground truncation, revealing that foreground truncation decreased as focal length decreased, but that this decrease did not account for the considerable expansion in distance perception.
\end{abstract}

The perception of distance in photographic displays has both theoretical and practical significance. Research in this area is beneficial to our understanding of pictorial perception and to our knowledge of general perceptual processes in the natural world (Gibson, 1971, 1979; Hagen, 1974; Kennedy, 1974). In terms of application, there is a growing need to design veridical and pedagogically effective visual simulations for training those skills that depend strongly on visual guidance (Haber, 1986). In all of these visual training simulations, from driver's education to the training of airline pilots, an appropriate representation of distance is critical. It is important, then, to examine systematically the nature of translating the natural visual world into the pictorial world.

One variable that may strongly influence our perception of distance in visual displays is the photographic area of view. Photographic area of view refers to the area of the scene before the camera that fits within the frame of the picture (Baker, 1980); it is a direct function of the focal length of the camera lens used to photograph the scene (Miller, 1972; Zettl, 1973). A "normal" viewing area is achieved by using a focal length whose circular image just covers the film onto which the image is projected: the focal length is equal to the diagonal of the

This paper was strengthened and clarified by the comments of several reviewers. Special thanks are due to John F. Patterson for his ideas on linear and power analyses and to Karen Thomson for her administrative support. Reprint requests should be sent to Robert N. Kraft, Psychology Department, Otterbein College, Westerville, OH 43081. rectangular film surface (Adams, 1970; Neblette \& Murray, 1973). With standard 35-mm photography, the normal lens has a focal length of approximately $48 \mathrm{~mm}$ and provides a photographic viewing area of approximately $50^{\circ}$. As focal length is shortened, from that of a normal lens to that of a wide-angle lens, the photographic area of view widens and the perceived distance of objects in the scene increases. As focal length is increased, from that of a normal lens to that of a telephoto lens, the photographic area of view becomes narrower and distance perception is compressed. The pictorial transformation is straightforward: focal length is directly proportional to image magnification and inversely proportional to the area of view. For example, doubling the focal length doubles the image magnification and halves the photographic area of view (Baker, 1980). However, the perceptual problem extends beyond the simple pictorial transformation. Differences in photographic distance perception arise from the fact that the area of view is limited to the borders of the projected image (Adams, 1970).

The effects of lens focal length are clearly illustrated in the aesthetic literature on photography and film (e.g., Adams, 1980; Coynik, 1974; Giannetti, 1987; Zettl, 1973), and the geometry of magnification and minification has been delineated in the psychological literature on picture perception (e.g., Lumsden, 1980, 1983; Rosinski \& Farber, 1980). What is lacking, however, is direct empirical validation: How do changes in lens focal length influence the perceptual experience of human observers as they judge distance in photographic displays? 
In a preliminary study, Kraft, Patterson, and Mitchell (1986) investigated the effects of widening the area of view of photographic displays from the normal perspective out to the widest possible rectilinear display. They did not examine the effects of compressing the area of view from the normal perspective into the narrow, telephoto range. Specifically, Kraft et al. examined the effects of four focal lengths $(48,28,24$, and $17 \mathrm{~mm}$ ) on perceived distances to naturally occurring target objects (e.g., trees) depicted in color slides of outdoor scenes. The subjects in this study were instructed to imagine themselves in the depicted scene and to judge how far away they were from particular target objects. As predicted in the aesthetic literature on photography, distance perception along the depth plane was systematically transformed by means of changing lens focal length; the shorter the focal length of the camera lens, the greater the perceived distance of objects in the scene. One issue that this study did not address is the source of these effects. What accounts for the increase in perceived distance as a function of decreasing lens focal length?

One component of the focal length effect may involve the extent of truncation in the visual field (Hagen, Jones, \& Reed, 1978). That is, as focal length decreases, there is less truncation of the visual field; in particular, more of the scene's foreground becomes visible to the viewer, toward whom the proximal edge of the pictorial visual field moves closer. The viewer then perceives the change in the edge of the pictorial visual field as a change in camera position and compensates by adjusting estimations of distance. In a pioneering study, Hagen et al. demonstrated the importance of truncation in compressing distance functions in a variety of delimited viewing conditions. In particular, they demonstrated that pictorial truncation will lead to compressed distance functions with standard photographic slides. However, this study did not include systematic manipulation of the extent of photographic truncation.

From this limited experimental literature on lens focal length, a number of questions emerge. What are the effects of compressing the photographic area of view from the normal into the telephoto range? How does the shape of the distance function change as focal length is systematically altered? Finally, what is the role of pictorial truncation in mediating these effects? The present study extended research on photographic area of view in three general ways: (1) A full range of focal lengths was examined: from extreme telephoto $(135 \mathrm{~mm})$ to normal $(48 \mathrm{~mm})$ out to extreme wide angle $(17 \mathrm{~mm})$. (2) A comprehensive scaling analysis was conducted to assess the systematic changes in distance perception as a function of focal length. Both linear and power functions were fitted to each individual's distance functions. (3) An initial test was conducted to assess the influence of truncation as a function of focal length. In a separate manipulation, viewers directly estimated the amount of foreground truncation caused by successively changing the focal length of the camera lens. These direct estimates of truncation were then compared to the estimates of distance to target objects depicted in the scene. In all these judgments, the subjects used direct magnitude estimation.

\section{METHOD}

\section{Subjects}

Seventy men and women from the introductory psychology class at Grinnell College served as subjects. The subjects were evenly divided into five groups, and there were approximately equal numbers of men and women.

\section{Materials}

Standard 35-mm slides were taken of 10 different natural settings in central Iowa. The subjects were not familiar with the particular settings. Each location was photographed with lenses of five different focal lengths: $135,80,48,28$, and $17 \mathrm{~mm}$. The lenses were selected to provide a wide range of photographic perspectives: from extreme telephoto to the widest possible rectilinear display. Each location was photographed so that a naturally occurring target object appeared in the approximate center of the display. Five target distances were used: $20,40,80,160$, and $320 \mathrm{~m}$. In all, each distance was represented twice; there were 2 locations $\times 5$ distances $\times 5$ lenses, for a total of 50 experimental slides. In addition to the experimental slides, 2 control slides were photographed. These were slides of natural settings with central target objects, but of locations different from those in the experimental slides.

The subjects viewed the slides while seated in a darkened classroom. The slides were projected with a Kodak Carousel slide projector on a screen at the front of the room; the subjects were seated between 2 and $4 \mathrm{~m}$ from the screen. They recorded their judgments on prepared data sheets.

\section{Procedure}

The experiment was run in two parts. In the main part, the subjects estimated the apparent distance to specified target objects in the slides. Before presentation of the slides, the subjects were told to make their estimates of distance in yards and given two heuristics for judging yardage: (1) the length of an average stride is approximately 1 yard and (2) a football field is 100 yards long.

The subjects were then introduced to the task of estimating pictorial distance with the two control slides. Each subject viewed all the experimental slides. As each slide was presented, the experimenter pointed out the target object. The subjects were told to put themselves "in the scene, taking the picture," and to judge the target object's apparent distance from their own positions in the scene. They were explicitly told to judge each slide as it was shown, independently of the other slides. The subjects were given as much time as necessary to make their judgments before the next slide was presented. The judgments were recorded by each subject on prepared data sheets. This procedure was repeated until all the slides had been presented.

Part 2 was run in the same session, after Part 1 . An identical set of slides was used in Part 2, but they were arranged in a different order. Sequences were presented that consisted of the five slides for any given location, ordered by decreasing focal length. The experimenters explained the manipulation of focal length to the subjects and then instructed them to focus on the foreground of each pictorial display. The subjects were then shown the initial slide in each sequence (the $135-\mathrm{mm}$ shot), and after presentation of the following slide (the $80-\mathrm{mm}$ shot), they were instructed to estimate, in yards, the amount of foreground that was revealed as they "moved back" in the scene. This procedure was repeated for the remaining slides in the sequence; each time, the subjects were required to estimate the apparent change in foreground distance with reference to the previously presented slide. This procedure was followed for 
all 10 sequences of slides. As before, the subjects recorded their judgments on prepared answer forms.

\section{Design}

Each subject saw all 50 slides. In Part 1, sequence and order effects were controlled for by means of a counterbalanced design for distance, focal length, and scene. The presentation order of the slides was determined by two balanced Greco-Latin square designs (Edwards, 1972; Winer, 1971). In addition, to eliminate effects of familiarity for any given location, the slides were arranged in five sets, with each location represented once in each set. The slides were presented in five different orders in which the sequences of the five sets were varied: (1) Sets 1, 2, 3, 4, 5; (2) Sets 2, 3, 4, 5, 1; (3) Sets 3, 4, 5, 1, 2; (4) Sets 4, 5, 1, 2, 3; (5) Sets 5, 1, $2,3,4$. The subjects were evenly divided across the five sequences. That is, for any given location, one-fifth of the subjects saw the extreme wide-angle perspective (17-mm focal length) first, one-fifth saw the wide-angle perspective $(28 \mathrm{~mm})$ first, one-fifth saw the normal perspective $(48 \mathrm{~mm}$ ) first, one-fifth saw the telephoto perspective $(80 \mathrm{~mm})$ first, and one-fifth saw the extreme telephoto perspective $(135 \mathrm{~mm})$ first. Thus, the subjects were run in five separate groups, with the starting point for each sequence of slides being different for each group. Viewing position was controlled through having each group of subjects occupy the same positions relative to the projection screen. The primary analysis for Part 1 was a oneway within-subjects analysis of variance with five levels of focal length. In addition, linear scaling functions relating judged to actual distance were determined with the method of least squares; power functions were fit by the method of least squares on the logarithms of judged and actual distance.

In Part 2, the subjects again viewed all 50 slides. The slides were organized in 10 sets of 5 slides, with each set containing the five focal length shots for a given scene. The sets were presented in order of increasing distance, and the slides within each set were presented in order of decreasing focal length. The direct estimates of foreground truncation from Part 2 were compared with the judged distances in Part 1. Specifically, computed differences were calculated from data obtained in Part 1 through subtraction of the judgments obtained for adjacent lenses at the same location and distance. These data were then compared against the corresponding judged differences in Part 2 with a 5 (distances) $\times 4$ (lens changes) withinsubjects analysis of variance.

\section{RESULTS}

Figure 1 shows judged distance as a function of actual distance for the five lens focal lengths. As predicted, photographic area of view significantly influenced distance perception along the depth plane $[F(4,276)=224.38$, $M S e=5,563.15, p<.001)$. As the focal length decreased and the area of view widened, distance judgments from observer to target object increased correspondingly. Duncan post hoc comparisons showed that each successive increase in focal length led to significantly greater perceived distances $(p<.05)$. The five slides for a given location were photographed at precisely the same station point, differing only with respect to the focal length of the lens used to take the slides. The differences in perceived distance are attributable solely to the pictorial transformations produced by changing the lens focal length.

For purposes of clarity, error bars have not been presented in Figure 1. For each focal length, the standard error for the distance judgments increased as a function of actual distance. The ranges of standard error from the shortest distance $(20 \mathrm{~m})$ to the longest distance $(320 \mathrm{~m})$ for each of the focal lengths were as follows: for the 17$\mathrm{mm}$ focal length, the range was 2.26 to 26.97 ; for the $28-\mathrm{mm}$ focal length, the range was 1.29 to 18.19 ; for the $48-\mathrm{mm}$ focal length, the range was 1.22 to 13.44 ; for the $80-\mathrm{mm}$ focal length, the range was .68 to 8.87 ; for the $135-\mathrm{mm}$ focal length, the range was .37 to 6.08 .

For the focal lengths from normal to telephoto, with one exception, judged distance was less than actual distance. For the wide-angle focal lengths, with one exception, judged distance was greater than actual distance. The telephoto view led to consistent underestimation of actual distance, whereas the wide-angle view led to consistent overestimation. Although the normal view generally engendered underestimation of actual distance, over the near range of distances-between 0 and $100 \mathrm{~m}$, distance perception was nearly veridical. The following scaling analyses explored more precisely the nature of these pictorial distance functions.

\section{Judged Distance as a Linear Function of Actual Distance}

Linear functions were fitted to each subject's distance judgments by the method of least squares. Table 1 shows the mean $y$-intercepts and slopes, along with the coefficients of determination for the linear distance functions. Both the $y$-intercepts and the slopes show a consistent increase as focal length decreases. Analyses of variance on the individual intercepts and slopes revealed highly significant effects: for the intercepts, $F(4,66)=46.73, M S \mathrm{e}$ $=395.21, p<.001$; for the slopes, $F(4,66)=96.66$, $M S e=.07, p<.001$. Duncan post hoc comparisons showed that all the slopes were significantly different and that all the intercepts except those for the two telephoto lenses were significantly different $(p<.05)$. There is a clear and consistent change in the distance functions as the photographic area of view is widened; the perceived distance expands and the perceived station point of the photograph moves closer to the viewer.

\section{Judged Distance as a Power Function of Actual Distance}

Log judged distance was related to log actual distance, in order to fit power functions to each subject's distance judgments. The slope of the resulting linear function is the exponent of a power function: given log (judged distance) $=\log K+e * \log$ (actual distance), then judged

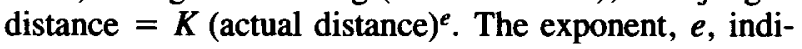
cates the curvature of the power function. Where $e=1$, perceived distance increases as a linear function of actual distance; where $e<1$, there is a negatively accelerating distance function indicating underconstancy; where $e>1$, there is a positively accelerating distance function indicating overconstancy.

Table 2 shows the mean and the standard deviation of the distributions of individual exponents, as well as the coefficient of determination, for each focal length. Two first-order generalizations can be made: (1) all focal 


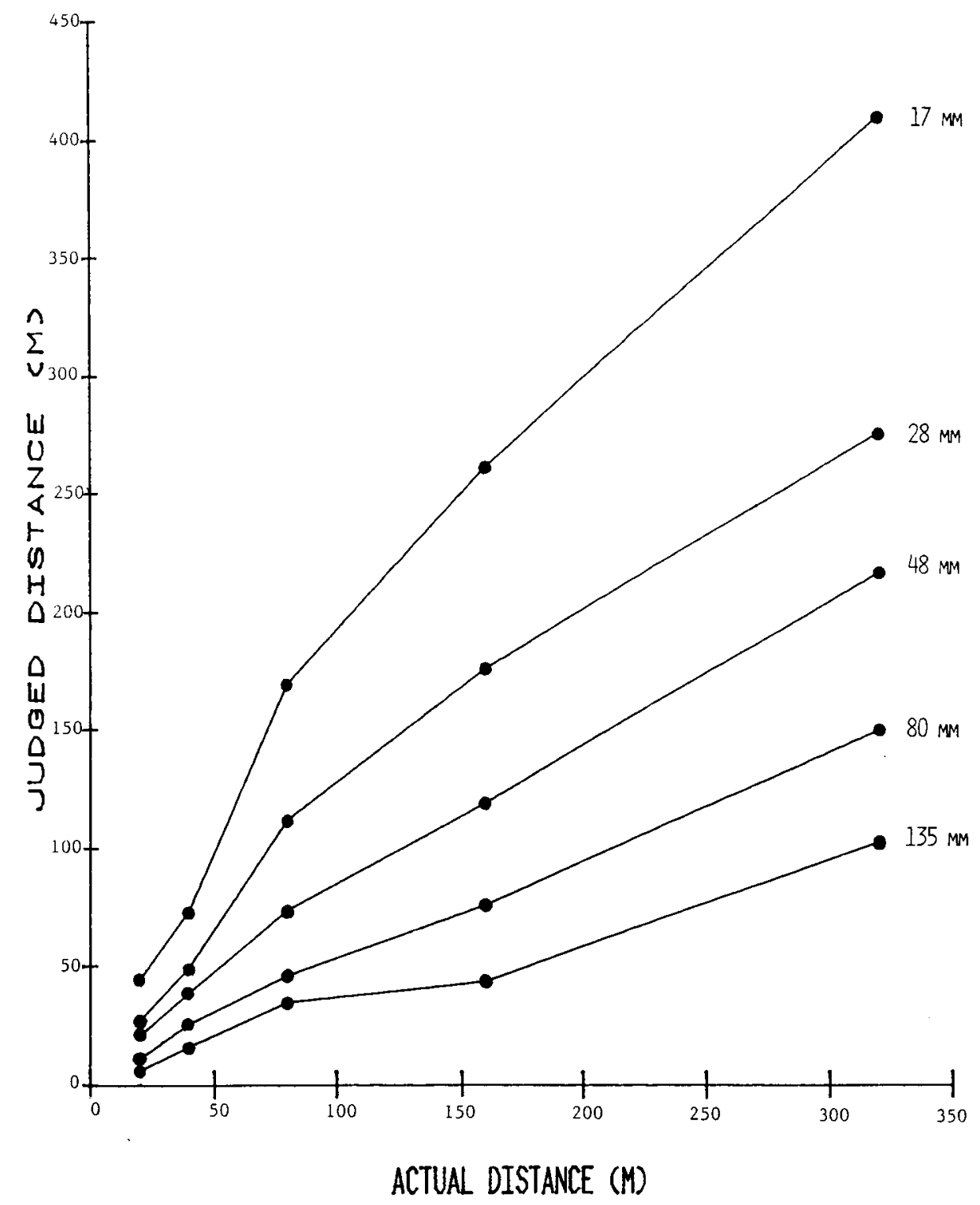

Figure 1. Judged distance as a function of actual distance in photographic slides taken with five different lens focal lengths: $17,28,48,80$, and $135 \mathrm{~mm}$.

lengths led to underconstancy, and (2) the exponents of the power functions generally decreased as a function of decreasing focal length. Perceived distance in photographic displays is a negatively accelerated function of actual distance, and the downward curvature of the distance function generally increases as focal length decreases and photographic area of view widens. An analysis of variance on the individual exponents showed a significant effect of focal length $[F(4,276)=12.46, M S e=.02$, $p<.001]$. Duncan post hoc tests revealed that there was a significant difference between the exponents for the telephoto views and the exponents for the normal to wideangle views $(p<.05)$.

The decrease in the exponent of the power function may be accounted for simply by the increase in the photo- graphic distance range (Da Silva, 1985; Teghtsoonian, 1973). That is, as focal length decreases, the photograph appears to present a greater range of distances, and the exponent of the power function decreases in response to this increasing stimulus range. Specifically, Teghtsoonian (1971) has shown that exponents are inversely propor-

Table 1

Linear Function Parameters for Judged Distance as a Function of Actual Distance for Each Focal Length (in mm)

\begin{tabular}{cccc}
\hline Focal Length & Slope & Intercept & $r^{2}$ \\
\hline 135 & .30 & 3.32 & .91 \\
80 & .45 & 6.38 & .93 \\
48 & .64 & 14.87 & .92 \\
28 & .81 & 27.30 & .88 \\
17 & 1.20 & 42.75 & .87 \\
\hline
\end{tabular}


Table 2

Mean Exponents, Standard Deviations, and Coefficients of Determination for Judged Distance as a Power Function of Actual Distance for Each Focal Length (in mm)

\begin{tabular}{cccc}
\hline & \multicolumn{3}{c}{ Exponent } \\
\cline { 2 - 4 } Focal Length & Mean & SD & $r^{2}$ \\
\hline 135 & .92 & .19 & .92 \\
80 & .89 & .18 & .93 \\
48 & .81 & .19 & .94 \\
28 & .82 & .20 & .92 \\
17 & .79 & .20 & .92 \\
\hline
\end{tabular}

tional to $\log$ stimulus ranges. Given this relationship, the decrease in exponents as a function of decreasing lens focal length can be taken as evidence for an actual increase in the range of photographic distance.

A $t$ test was conducted for each focal length, to compare the $r^{2}$ values for the linear fit as opposed to the power fit. Significant differences were found in the normal to wide-angle range [for the $48-\mathrm{mm}$ focal length, $t(68)=$ $2.23, p<.05$; for the $28-\mathrm{mm}$ focal length, $t(68)=2.28$, $p<.05$; for the $17-\mathrm{mm}$ focal length, $t(68)=3.35$, $p<.01]$. There were no significant differences in $r^{2}$ for the two telephoto lenses. Power functions showed a small but significant improvement over linear functions in the normal to wide-angle range, but no improvement at all in the telephoto range.

\section{Foreground Truncation}

In Part 2, the subjects directly estimated the amount of foreground that was revealed as the photographic area of view of a given scene was successively widened. That is, they attended to the foreground of each depicted scene and provided direct estimates, in yards, of how much foreground came into view as the lens focal length changed from 135 to 80 to 48 to 28 to $17 \mathrm{~mm}$. These estimates provided a relative measure of foreground truncation as a function of lens focal length. The critical comparison here is between the perceived increase in the distance to the target object (Part 1) and the directly estimated change in foreground truncation (Part 2) as a function of successively decreasing focal length. This comparison is shown graphically in Figure 2. Significant differences were found for lens change $[F(3,201)=44.32, M S e=$ $1,277.35, p<.001]$ and type of judgment $[F(1,67)=$ $184.72, M S e=3,469.69, p<.001]$. The interaction was

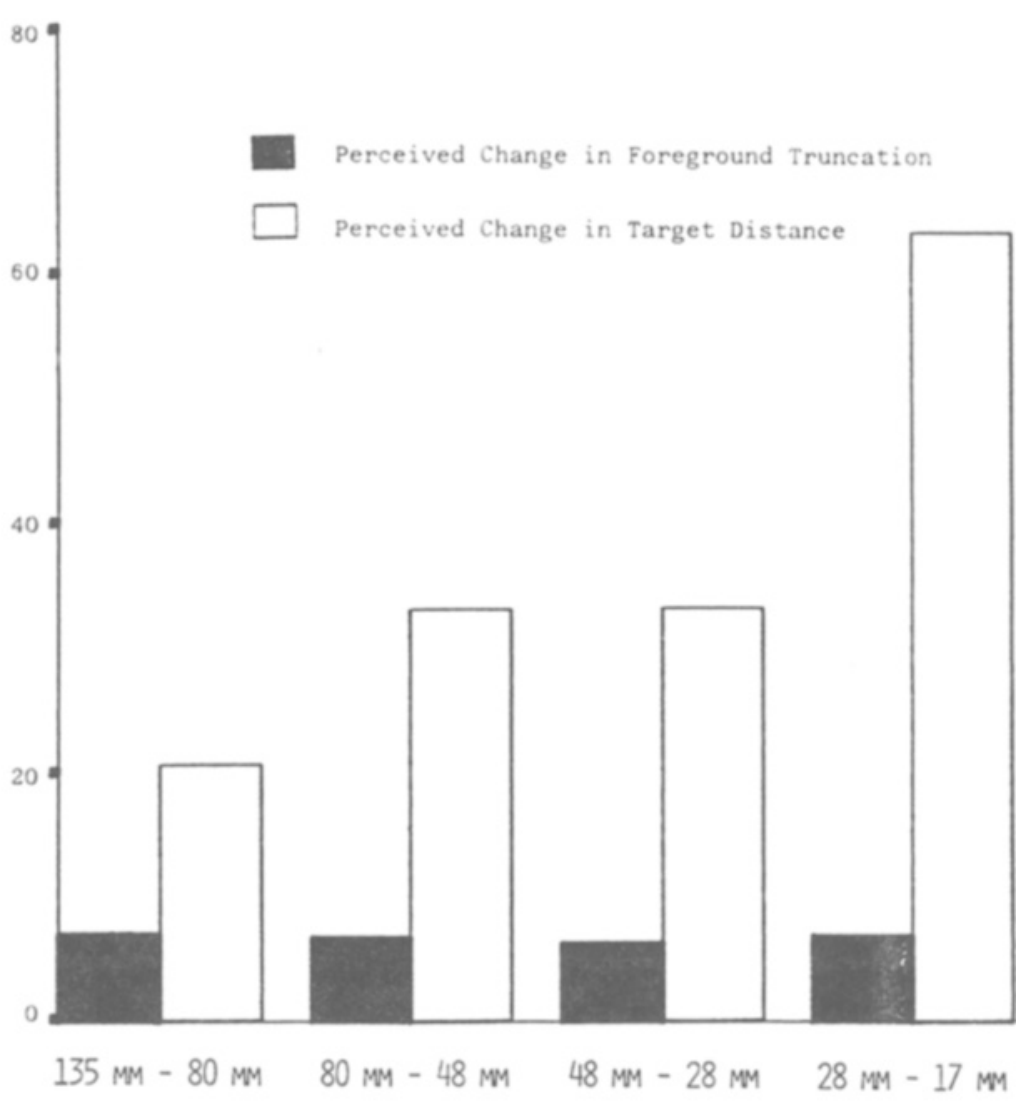

CHWGE N LES FOCN DENTH

Figure 2. Perceived increase in target distance and estimated change in foreground truncation as a function of successively decreasing lens focal length. 
also significant $[F(3,201)=44.39, M S \mathrm{e}=1,268.47$, $p<.001$ ], indicating that successive decreases in lens focal length had differential effects on perceived distance and judged truncation.

The change in perceived distance to target objects grew substantially as a function of successively decreasing lens focal length, whereas the perceived change in foreground truncation remained relatively constant. As focal length decreased and the area of view widened, the amount of expansion in the perceived distance to target objects not explainable by truncation showed a consistent increase (see Figure 2). Given an unspecified amount of truncation in the visual field, viewers seem to apply a small fixed value to all of their distance judgments (Hagen et al., 1978). Changes in foreground truncation accounted for a constant amount of change in perceived distance, but as focal length was shortened, other features of the photographic display played an increasingly larger role in the perception of distance.

Judgments of foreground truncation can be compared to the actual changes in the amount of depicted foreground as a function of focal length. For each lens focal length, the distance was measured from the camera to the closest visible point in the center of the foreground. When projected, this point was at the center of the bottom edge - the proximal edge - of the photographed scene. The 35-mm edge of the film was horizontal, and the horizon bisected the upper and lower halves of the picture frame.

The total amount of foreground visible in the picture frame increased as focal length decreased and area of view widened. The center of the foreground edge was $17.47 \mathrm{~m}$ from the camera position in the $135-\mathrm{mm}$ shot and $2.36 \mathrm{~m}$ from the camera position in the $17-\mathrm{mm}$ shot. The change in visible foreground with each successive change in focal length decreased as focal length decreased. For the change in focal length from 135 to $80 \mathrm{~mm}$, the center of the foreground edge moved $6.93 \mathrm{~m}$ closer to the camera; for the change from 80 to $48 \mathrm{~mm}$, the foreground edge moved $4.18 \mathrm{~m}$ closer; for the change from 48 to $28 \mathrm{~mm}$, the foreground edge moved $2.51 \mathrm{~m}$ closer; for the change from 28 to $17 \mathrm{~mm}$, the foreground edge moved $1.49 \mathrm{~m}$ closer. The actual changes in the visible foreground stand in contrast to the subjects' judgments; the actual change decreased by a factor of .6 with each successive change in lens focal length, whereas the subjects' judgments remained relatively constant (Figure 2 ).

\section{DISCUSSION}

The perception of photographs was examined under natural viewing conditions: binocular viewing with an unconstrained field of view and free head movement. The perceived distance to target objects along the pictorial depth plane was systematically transformed by means of changes in the photographic area of view: the shorter the focal length of the camera lens, the wider the area of view, and the greater the perceived distance. Focal length showed a strong and consistent effect from extreme telephoto $(135 \mathrm{~mm})$ out to extreme wide angle $(17 \mathrm{~mm})$. Specifically, significant differences were found for all five focal lengths represented in this study: $135,80,48,28$, and $17 \mathrm{~mm}$. Since all five slides of the given scenes were photographed at precisely the same station point, the differences in perceived distance are attributable solely to the pictorial transformation produced by changing the lens focal length.

Viewing position was controlled in this study by means of having each experimental group occupy the same position relative to the projection screen. Within each group, the individual subjects occupied different positions relative to the projection screen. It can be hypothesized that appreciable variation in distance estimates was introduced by the subjects' different positions. However, such increases in variability are unlikely, given the conditions under which the photographs were viewed: binocular viewing with an unconstrained field of view and free head movement. Under these conditions, the perception of pictures is not influenced by changes in viewing position (Neblette \& Murray, 1973). More specifically, with unconstrained binocular viewing, displacements from the geometrically correct station point do not affect observers' perception of pictorial perspective (e.g., Gibson, 1979; Yonas \& Cooper, 1976). Moreover, the pattern of data indicates an orderly, systematic change in perceived distance as a function of focal length.

Linear functions were fitted to each subject's distance judgments, revealing a very high goodness of fit. Both the $y$-intercepts and the slopes increased as the focal length decreased. The increasing $y$-intercepts suggest that viewers place themselves farther away from the depicted objects as the focal length decreases, compensating for the advancing proximal edge. More direct support for this notion was provided in Part 2 of this study. In Part 2, the subjects directly estimated the change in truncation of the pictorial foreground. As the focal length was shortened, the subjects reported a corresponding decrease in the amount of foreground truncation. The amount of change in truncation grew in fixed steps, however, and did not account for the markedly steep increase in perceived target distance as a function of focal length. Given pictorial truncation, viewers seem to apply a small fixed value to all of their distance judgments (Hagen et al., 1978). The increasing slopes in the linear functions provide further support for this observation, indicating that distance information throughout the depth plane appeared to change as the focal length was systematically transformed.

Power functions were fitted to each subject's distance judgments. In the normal to wide-angle range, the power functions provided slightly better fits than the linear functions, reflecting the downward concavity of the distance functions with the wider photographic perspectives. As the focal length was shortened and the photographic display became less truncated and more expansive-more similar to a natural viewing situation-curvilinearity increased, and the exponential model provided a more accurate description of the data. In the telephoto range, 
power functions and linear functions provided equivalent fits. Given these findings, it seems appropriate to discuss the relative merits of linear as opposed to exponential models. Unfortunately, the choice between linear functions and power functions is not a straightforward one. When Hagen and Teghtsoonian (1981) compared linear and power functions in their investigation of binocular and motion-generated information in a nonphotographic setting, power functions more accurately described the subjects' judgments of depth for monocular viewing but not for binocular viewing. The overall pattern of results obtained with linear and exponential models was the same across all conditions. The major argument in favor of exponential modeling was that it accounted for the curvilinearity in the distance judgments of individual subjects. On the basis of our results, however, a stronger case can be advanced to support linear modeling for judgments of photographic distance. In general, linear modeling presents a more elegant approach. Moreover, the rather large increase in descriptive power of an exponential model does not seem to be justified by the results of this study. The wide range of distances represented in this study (20 to $320 \mathrm{~m}$ ), the high values of $r^{2}$ with linear functions, and the small increase in goodness of fit with power functions argue in favor of modeling these photographic distance judgments with the simpler linear form.

In general, the influence of focal length on photographic distance perception moves beyond the straightforward pictorial transformations. Differences in photographic distance perception occur because the area of view is limited by the borders of the photographic display. Perspective per se is not distorted by changes in lens focal length (Adams, 1970; Baker, 1980). For example, given a constant camera position, if the entire photograph produced by a telephoto lens were reduced in size by the appropriate factor, it would exactly match the center portion of a photograph produced by a normal lens. Conversely, with camera position held constant, if the center portion of a wide-angle photograph were removed from the photograph and increased in size by the appropriate factor, it would exactly match the entire display produced by a normal lens (Adams, 1970; Baker, 1980).

Perceptually, the difference between a telephoto view and a normal view lies in the observer's being restricted to the borders of the photographic display. Because of this restriction, the telephoto image appears to be magnified (Adams, 1970). In the natural environment, the visual field begins at the face (Hagen et al., 1978). With a photograph, however, the scene begins some distance away from the observer, and the observer makes an adjustment. This adjustment then changes as a function of the photographic area of view. For example, as the focal length decreases, the area of view widens, more of the foreground becomes visible, and depicted objects in the scene become smaller. Observers then adjust their position within the scene, placing themselves farther away and increasing their estimates of distance. The increasing $y$ intercepts in the linear functions indicate that observers are placing themselves farther away from the depicted objects as focal length is shortened.

In terms of praxis, these results may be applied to the design and development of systems that support visually guided behavior or visual monitoring (Stewart, Middleton, Downton, \& Ely, 1984). With such systems, it is often desirable to present visual displays with the widest possible area of view, allowing the user to scan the simulated environment more naturally (Patterson, Buede, Kraft, \& Mitchell, 1982). However, widening the area of view by shortening the focal length of the lens alters the user's perception of distance. Photographic area of view, then, is an important consideration in the formatting of visual displays.

\section{REFERENCES}

Adams, A. (1970). Camera and lens. Hastings-on-Hudson, NY: Morgan \& Morgan.

Adams, A. (1980). The camera. Boston: Little, Brown.

BAKER, A. A. (1980). Photography: Art and technique. San Francisco: W. H. Freeman.

CoYNIK, D. (1974). Movie making. Chicago: Loyola University Press. Da Silva, J. A. (1985). Scales for perceived egocentric distance in a large open field: Comparison of three psychophysical methods. American Journal of Psychology, 98, 119-144.

EDWARDS, A. L. (1972). Experimental design in psychological research. New York: Holt, Rinehart \& Winston.

GiannetTi, L. D. (1987). Understanding movies. Englewood Cliffs, NJ: Prentice-Hall.

Gibson, J. J. (1971). The information available in pictures. Leonardo, 4, 27-35.

GiBson, J. J. (1979). The ecological approach to visual perception. Boston: Houghton Mifflin.

HABER, R. N. (1986). Flight simulation. Scientific American, 255(1), 96-103.

HAGEN, M. A. (1974). Picture perception: Toward a theoretical model. Psychological Bulletin, 81, 471-497.

HAGEN, M. A., Jones, R. K., \& ReED, E. S. (1978). On a neglected variable in theories of pictorial perception: Truncation of the visual field. Perception \& Psychophysics, 23, 326-330.

Hagen, M. A., \& Teghtsoonian, M. (1981). The effects of binocular and motion-generated information on the perception of depth and height. Perception \& Psychophysics, 30, 257-265.

KENNEDY, J. M. (1974). A psychology of picture perception. San Francisco: Josey-Bass.

Kraft, R. N., Patterson, J. F., \& Mitchell, N. B. (1986). Distance perception in photographic displays of natural settings. Perceptual \& Motor Skills, 62, 179-186.

LUMSDEN, E. A. (1980). Problems of magnification and minification: An explanation of the distortions of distance, slant, shape, and velocity. In M. A. Hagen (Ed.), The perception of pictures: Vol. 1. Alberti's window: The projective model of pictorial information (pp. 91135). New York: Academic Press.

LUMSDEN, E. A. (1983). Perception of radial distance as a function of magnification and truncation of depicted spatial layout. Perception \& Psychophysics, 33, 177-182.

Miller, F. (1972). College physics. New York: Harcourt Brace Jovanovich.

Neblette, C. B., \& Murray, A. E. (1973). Photographic lenses. Dobbs Ferry, NY: Morgan \& Morgan.

Patterson, J. F., Buede, D. M., Kraft, R. N., \& Mitchell, N. B. (1982). An investigation of the feasibility for implementing an advanced terrain representation system. McLean, VA: Decisions and Designs, Inc.

RoSINSKI, R. R., \& FARBER, J. (1980). Compensation for viewing point in the perception of pictured space. In M. A. Hagen (Ed.), The per- 
ception of pictures: Vol. 1. Alberti's window: The projective model of pictorial information (pp. 137-176). New York: Academic Press.

Stewart, T. R., Middleton, P., Downton, M., \& Ely, D. (1984). Judgements of photographs vs. field observations in studies of perception of the visual environment. Journal of Environmental Psychology, 4, 283-302.

Teghtsoonian, R. (1971). On the exponents in Stevens' law and the constant in Ekman's law. Psychological Review, 78, 71-80.

TEGHTSOONIAN, R. (1973). Range effects in psychophysical scaling and a revision of Stevens' law. American Joumal of Psychology, 86, 3-27.
WiNER, B. J. (1971). Statistical principles in experimental design. New York: McGraw-Hill.

YonAS, A., \& COOPER, R. (1976). Report on the 1975 conference on pictorial perception at the University of Minnesota, U.S.A. Leonardo, 9, 311-313.

ZeTTL, H. (1973). Sight sound motion: Applied media aesthetics. Belmont, CA: Wadsworth.

(Manuscript received July 18, 1986;

revision accepted for publication November 8,1988 .) 